\title{
New Instrument Concepts for Ocean Sensing: Analysis of the PAU-Radiometer
}

\author{
Adriano Camps, Senior Member, IEEE, Xavi Bosch-Lluis, Isaac Ramos-Pérez, Associate Member, IEEE, \\ Juan Fernando Marchán-Hernández, Student Member, IEEE, Benjamín Izquierdo, \\ and Nereida Rodríguez-Álvarez, Student Member, IEEE
}

\begin{abstract}
Sea surface salinity can be remotely measured by means of L-band microwave radiometry. However, the brightness temperature also depends on the sea surface temperature and on the sea state, which is probably today one of the driving factors in the salinity retrieval error budgets of the European Space Agency's Soil Moisture and Ocean Salinity (SMOS) mission and the NASA-Comisión Nacional de Actividades Espaciales Aquarius/SAC-D mission. This paper describes the Passive Advanced Unit (PAU) for ocean monitoring. PAU combines in a single instrument three different sensors: an L-band radiometer with digital beamforming (DBF) (PAU-RAD) to measure the brightness temperature of the sea at different incidence angles simultaneously, a global positioning system (GPS) reflectometer [PAU-reflectometer of Global Navigation Satellite Signals (GNSS-R)] also with DBF to measure the sea state from the delay-Doppler maps, and two infrared radiometers to provide sea surface temperature estimates. The key characteristic of this instrument is that both PAU-RAD and the PAU-GNSS/R share completely the RF/IF front-end, and analog-to-digital converters. Since in order to track the GPS-reflected signal, it is not possible to chop the antenna signal as in a Dicke radiometer, a new radiometer topology has been devised which makes uses of two receiving chains and a correlator, which has the additional advantage that both PAU-RAD and PAU-GNSS/R can be operated continuously and simultaneously to perform the sea-state corrections of the brightness temperature. This paper presents the main characteristics of the different PAU subsystems, and analyzes in detail the PAU-radiometer concept.
\end{abstract}

Index Terms-Correlator, Global Navigation Satellite Signals (GNSS), radiometry, reflectometry, sea salinity, sensitivity.

\section{INTRODUCTION}

$\mathbf{I}$ T IS WELL known that sea surface salinity can be remotely measured by means of L-band microwave radiometry [1]. Currently, two spaceborne missions are planned to be launched in the near future with this purpose: the European Space Agency's Soil Moisture and Ocean Salinity (SMOS) mission [2], using a Y-shaped synthetic aperture radiometer, and NASA-Comisión Nacional de Actividades Espaciales AQUARIUS/SAC-D mission [3], using a tree-beam pushbroom radiometer.

Manuscript received September 28, 2006; revised December 12, 2006. This work was supported in part by the participating organizations of EURYI and in part by the EC Sixth Framework Programme.

The authors are with the Universitat Politècnica de Catalunya Campus Nord, 08034 Barcelona, Spain (e-mail: camps@tsc.upc.edu).

Color versions of one or more of the figures in this paper are available online at http://ieeexplore.ieee.org.

Digital Object Identifier 10.1109/TGRS.2007.894925
Sea surface salinity can be indirectly measured through the variations of the brightness temperature due to the change of the sea water dielectric constant with respect to temperature and salinity. However, the brightness temperature also depends on the sea surface roughness, which cannot be simply parameterized in terms of the wind speed, the significant wave height, or any other currently available parameter. Therefore, despite the field experiments performed in the past years to improve our understanding of this effect [4], the sea surface roughness correction still remains one of the most critical corrections needed to retrieve the salinity with the required accuracy, and inaccuracies in the brightness temperature direct model may induce significant errors (biases) in the retrieved salinity [5].

In the SMOS mission, the multiangle observation capabilities allow to simultaneously retrieve not only the surface salinity, but in addition, the surface temperature and an "effective" wind speed that minimizes the salinity retrieval error, as first proposed in [6]. In AQUARIUS, an L-band scatterometer will be used to perform the necessary sea-state corrections.

The potential use of reflectometry of Global Navigation Satellite Signals (GNSS-R) opportunity signals for altimetry [7] and sea-state determination in terms of the mean-square slope has been already tested from ground-based [8]-[11], airborne [12]-[19], and spaceborne [20], [21] experiments. However, the underlying science still needs further refinements to extract meaningful physical quantities that can be successfully used in the remote sensing and oceanographic communities. Probably, one of the main advantages of this technique lies in the capability to obtain simultaneous and collocated sea-state information, which is not possible with other auxiliary data sets [5]. A performance study of the capabilities and requirements of this technique applied to the SMOS case was recently presented [22].

The Passive Advanced Unit (PAU) is a new instrument concept that was proposed in 2003 to the European Science Foundation (ESF) within the frame of the European Young Investigator (EURYI) Awards program, and was funded in 2004 [23]. It consists of a suite of three instruments operating in a synergetic way: 1) PAU-RAD: an L-band radiometer to measure the brightness temperature of the sea surface; 2) PAU-GNSS/R: a reflectometer to measure the sea state using reflected global positioning system (GPS) opportunity signals; and 3) and PAU-IR: two infrared (IR) radiometers to measure the sea surface temperature.

The L-band radiometer is an array of $4 \times 4$ dual-polarization receivers [24] integrated behind a patch antenna, whose outputs 


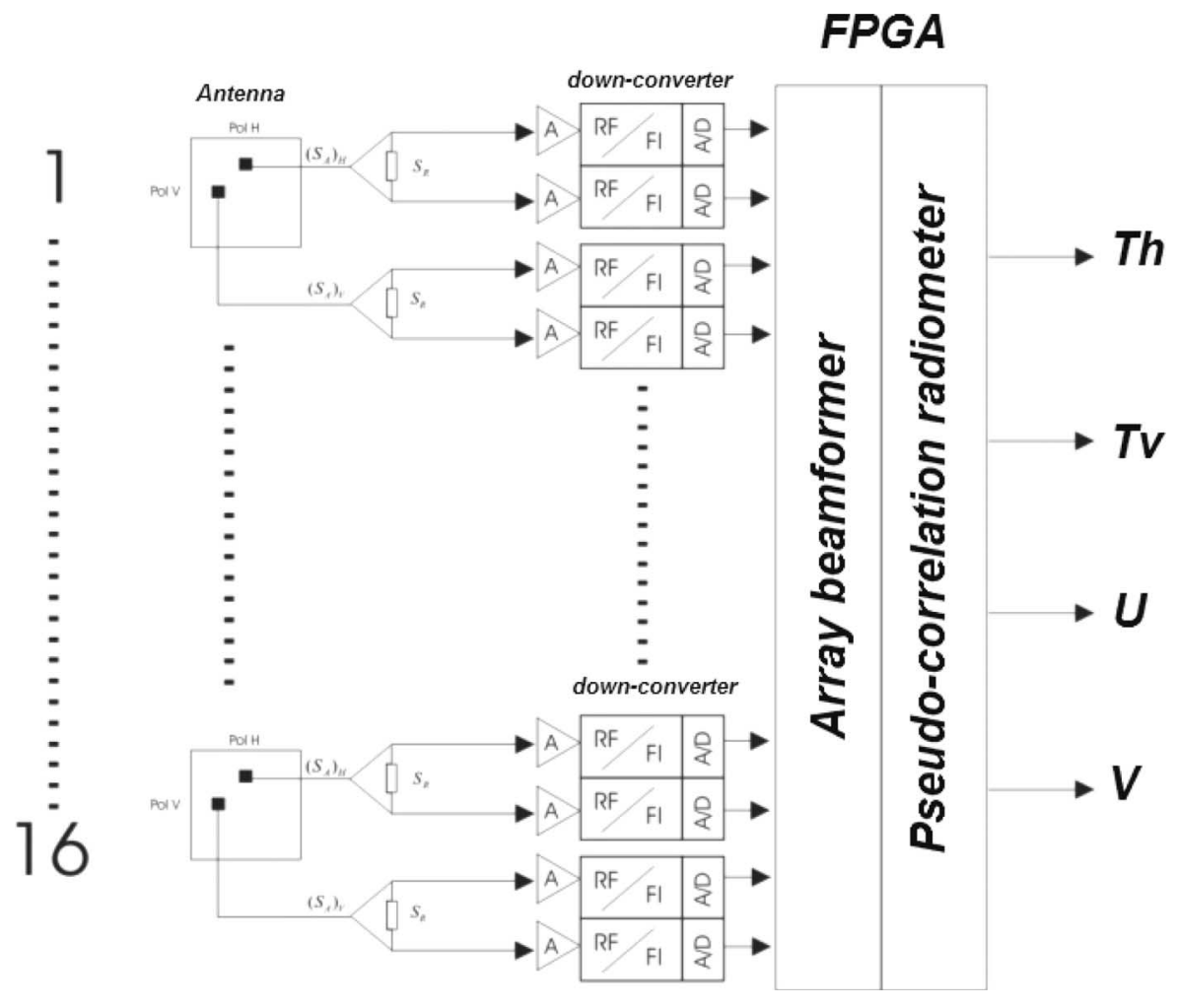

Fig. 1. Schematic of the PAU-RAD $4 \times 4$ array.

are digitized and then properly calibrated and combined to produce several beams using a digital beamformer (DBF) [25]. The GPS-reflectometer uses the same hardware as the L-band radiometer, but the DBF synthesizes beams pointing toward the specular reflection points of the GPS signals [26]. Finally, the third element is a pair of commercial $8-14-\mu \mathrm{m}$ thermal IR radiometers.

Section II describes the PAU instrument concept at system level. The detailed analysis of PAU-RAD using noise waves is presented in Section III for the general case, including imperfect Wilkinson isolation, imperfect matching, etc., and then simplified to the ideal case. Finally, Section IV presents the analysis of the predicted performance in terms of angular resolution and radiometric sensitivity.

\section{Overall Instrument Description}

\section{A. Receiver Front-End}

One of the technological goals of the PAU instrument is to demonstrate the feasibility of combining in a single receiver two types of instruments: the radiometer (PAU-RAD) which, for stability reasons, cannot be a total power radiometer, and the GPS-reflectometer (PAU-GNSS/R). In order to be able to use the same receivers for both the radiometer and the GPSreflectometer, a new radiometer topology has been devised (Fig. 1). To understand the concept, the ideal case is discussed here, leaving to Section III the general analysis using noise waves.

As compared to a real aperture radiometer, instead of connecting the antenna output directly to the radiometer receiver, each receiving element is connected to the input of a Wilkinson power splitter ${ }^{1}$ that divides the signal in two signals that are in phase. However, the $100-\Omega$ resistor of the Wilkinson power splitter that connects the two outputs also adds two noise signals that are $180^{\circ}$ out of phase. Therefore, the signals at the input of the two channels of the radiometer are the sum and the difference of the antenna signal and the noise generated by the Wilkinson power splitter resistor. Once properly amplified, down-converted, sampled, and combined with the ones from other receivers to digitally form the beam(s), they are finally cross-correlated leading to an output that is proportional to the difference between the antenna temperature and the physical temperature of the Wilkinson power splitter resistor. That is, the system output is the same as the one of the Dicke radiometer, but the input signal is not chopped, so that it can be used to track the GPS-reflected signal continuously and simultaneously with the radiometric measurements (Section III).

Two additional calibration signals are used for internal calibration purposes: uncorrelated noise, generated by a matched load at each input channel, to compensate for instrumental biases, and two levels of correlated noise from a common noise source, to compensate for phase and amplitude mismatches among receivers (Fig. 2, Section III-A). External calibration is performed using two external references, the hot/cold load technique using described for example in [33], with the sky as cold load.

\footnotetext{
${ }^{1}$ In its simplest form, a Wilkinson power splitter consists of two transmission lines of characteristic impedance equal to $\sqrt{2} Z_{0}$ connected at the input and connected through a $2 Z_{0}$ resistor at the outputs. All ports are perfectly matched, and the outputs are perfectly isolated. Any other three ports having the same S-parameter matrix will produce the same noise waves at its outputs. See for example:http://www.microwaves101.com/encyclopedia/Wilkinson_ splitters.cfm\#twoport.
} 


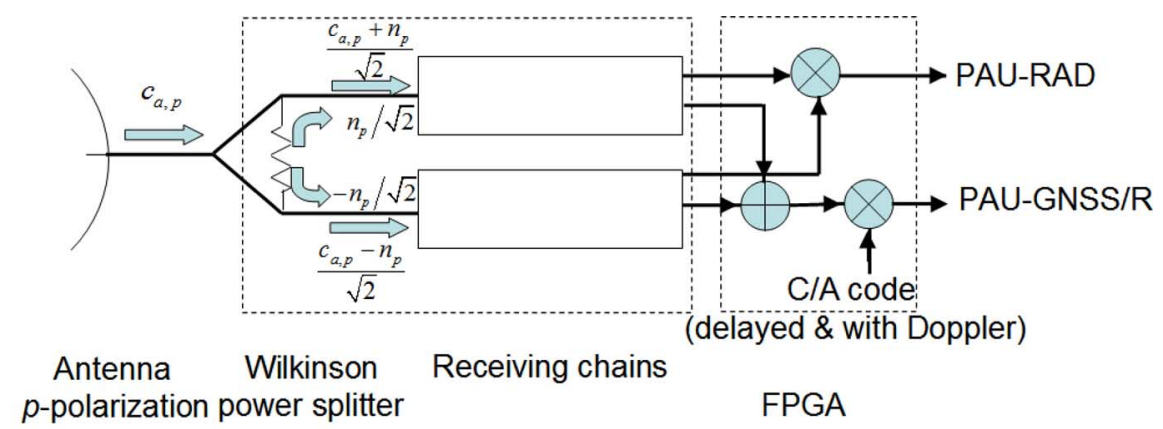

(a)

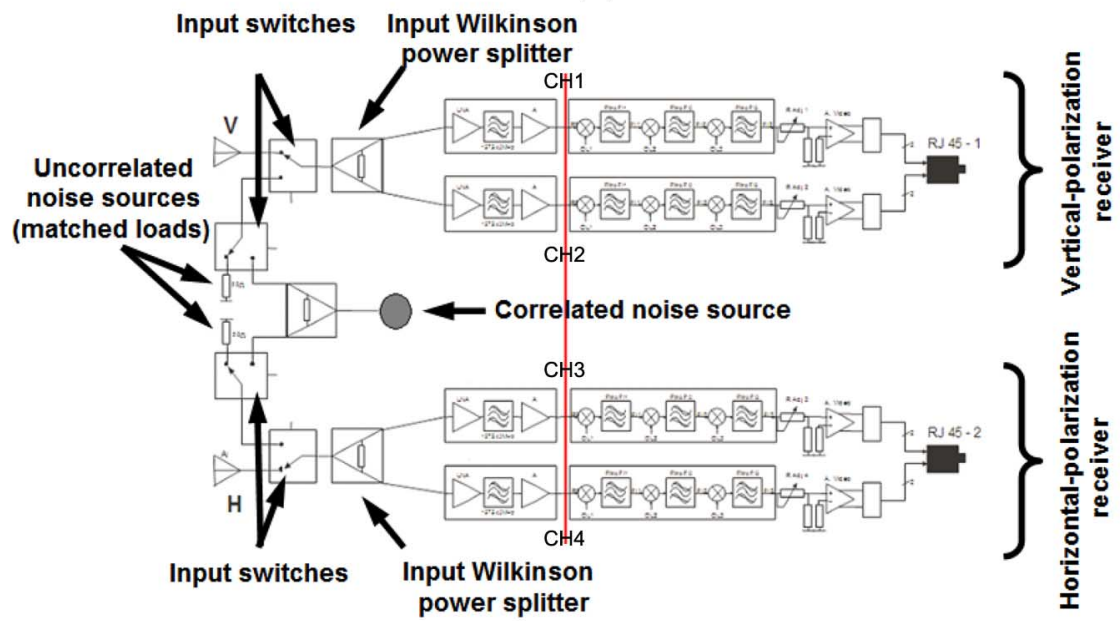

(b)

Fig. 2. (a) Concept block diagram of a one-receiver PAU-RAD. $c_{a, p}$ is the noise collected by the antenna (including the one generated by its ohmic losses), and $n_{p}$ is the noise generated by the Wilkinson power splitter resistor. (b) Receiver block diagram of the dual-polarization receivers for PAU-RAD.

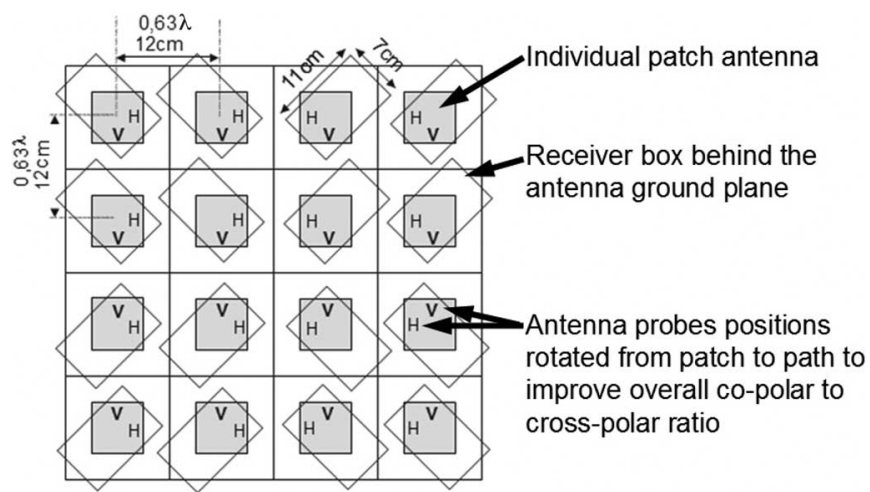

Fig. 3. Array of $4 \times 4$ elements pointing to the ocean. The array boresight will be $45^{\circ}$ off nadir.

The beam of the microwave radiometer was required to have a $20^{\circ}$ half-power beamwidth with a main beam efficiency (MBE) larger than $95 \%$ for pointing angles up to $\pm 20^{\circ}$ from the boresight direction, which will be pointed $45^{\circ}$ off nadir to cover the range of incidence angles from $25^{\circ}$ to $65^{\circ}$. These three requirements are satisfied with a $4 \times 4$ array of elements spaced 0.63 wavelengths (Fig. 3) at the GPS L1 frequency

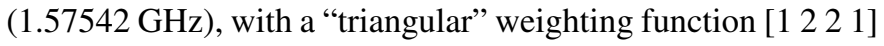
in both dimensions, that is $\left[\begin{array}{llll}1 & 2 & 2 & 1\end{array}\right]$ for the first and fourth rows, and [ $\left.\begin{array}{llll}2 & 4 & 4 & 2\end{array}\right]$ for the second and third rows.

The interelement spacing determines the maximum size of the receiver to which the antenna is connected. Three receivers are shown in Fig. 4: the elementary element antennas are

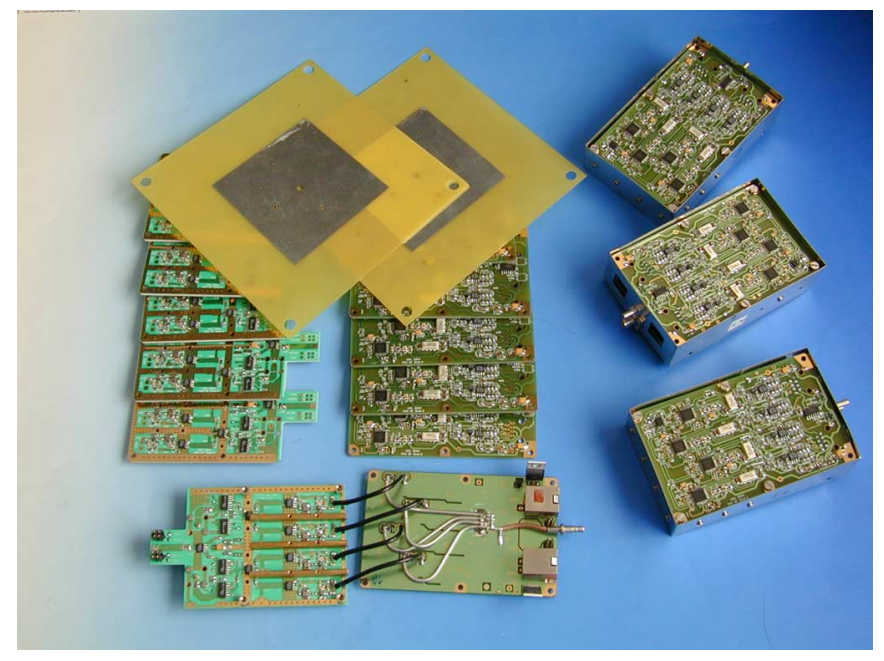

Fig. 4. Elementary element antennas microstrip patch antennas (top) and RF (left) and IF (center) boards that are interconnected by means of four semiflexible cables, folded, and integrated in a $3 \times 7 \times 11 \mathrm{~cm}$ box (right).

microstrip patch antennas, which are very convenient at this frequency (top), and the RF (left) and IF (center) boards, which are interconnected by means of four semiflexible cables, folded, and integrated in a $7 \times 11 \times 3 \mathrm{~cm}$ box (right).

The isolation between channels is better than $40 \mathrm{~dB}$, which is suitable for the measurement of the four Stokes parameters. The output IF signals are transmitted at a central frequency of 4.3 MHz through a pair of RJ-45 grade five cables and applying 


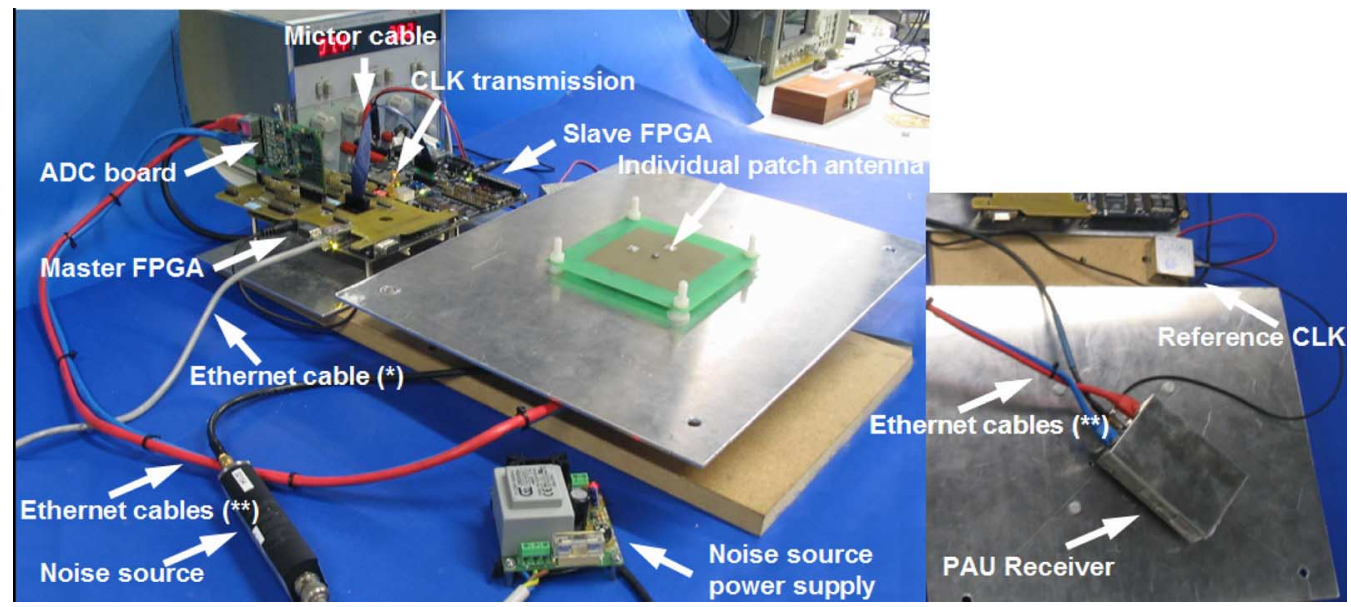

Fig. 5. Laboratory setup showing the different parts of the one-receiver version of PAU. Each receiver is integrated behind each individual dual-polarization patch antenna. All receivers are driven by the same 10-MHz reference clock and by a common noise source used for correlated noise injection. Receiver outputs are sent to the ADC board through a pair of Ethernet cables $\left(^{* *}\right)$. The four sampled channels $\left(f_{s}=5.745 \mathrm{MHz}\right)$ are time-multiplexed $(\times 4)$ before input to the master FPGA. Master and slave FPGAs operate synchronously with the same clock and exchange information through a high-speed Mictor cable. The data processing includes Stokes parameters computation and DDM generation, and in the $4 \times 4$ array the generation of the different beams for both the radiometer (PAU-RAD) and the reflectometer (PAU-GNSS/R). Final products are sent to the external world through an Ethernet link $\left(^{*}\right)$.

the bandpass sampling technique are down-converted and the in-phase and quadrature (I/Q) signals are obtained. ${ }^{2}$

\section{B. Analog-to-Digital Converters (ADCs)}

The eight-bit samplers are formed by an array of 16 ADC cards whose inputs are the outputs of each receiving unit: four channels per receiving unit. The throughput of each ADC card is: 4 channels $\times 8$ bits $\times 5.745 \mathrm{MHz}=183.84 \mathrm{Mb} / \mathrm{s}$, and the total input rate at the field programmable gate arrays (FPGAs) is thus 16 times higher, approximately $3 \mathrm{~Gb} / \mathrm{s}$. This amount of data enters into the two FPGAs by using nearly all available I/O pins, and by using time multiplexing by a factor 4 , that is four signals sampled at $5.745 \mathrm{MHz}$ are input at $22.98 \mathrm{MHz}$.

\section{DBF Processor}

The design is split in two parts and fit in two Altera FPGAs Stratix EP1S10F780C6E to implement PAU-RAD using the very high-speed integrated circuit hardware description language (VHDL)-93 digital description language. The first one is called the control unit and manages the system. The control unit has an external communications module using the TCP/IP protocol in order to provide a standard interface to remote control the system. Furthermore, it calculates the corrections to be applied to the digital I/Q signals for calibration. These functions have been developed using the Altera VHDL Nios microprocessor, which makes the design of the control module easier and more versatile, since it is programmed using ANSI-C. The second part of the system is called arithmeticlogic unit, where the algorithm is implemented. Signals are dig-

\footnotetext{
${ }^{2}$ The bandpass sampling technique, or IF or RF sampling, refers to a technique in which a signal of bandwidth $B$, centered at $f_{0}$ is sampled without loss of information at a frequency $f_{s}$ much smaller than $2 \cdot\left(f_{0}+B / 2\right)$, which is given by: $2(B+(n B+r) /(N-n+1)) \leq f_{s} \leq 2(B+(n B+$ $r) /(N-n))$ with $N<\left(f_{0}-B / 2\right) / B<N+1<\left(f_{0}+B / 2\right) / B, r=$ $f_{0}-(B / 2)-N \cdot B$, and $n=0, \ldots, N-1$ (http://en.wikipedia.org/wiki/ Sampling_\%28signal_processing\%29\#IF.2FRF_sampling).
}

itally processed in this block to obtain the four Stokes parameters with maximum accuracy. The PAU-RAD design includes other VHDL blocks as well that are not directly related to the radiometer subsystem but to the PAU-GNSS/R reflectometer.

\section{Real-Time Delay-Doppler Map (DDM) Generator}

To generate a DDM from a stream of GPS data downconverted and sampled it is necessary to generate simultaneously several different frequencies along with a number of shifted versions of the corresponding pseudorandom-noise (PRN) code. To obtain real-time DDM waveforms a DDM generator has been implemented in an FPGA using VHDL digital logic description language. To simplify the system, a commercial GPS receiver is added to the system to provide some "a priori" information on which satellites are visible and which are their respective delay and Doppler offsets with respect to the direct signals. This information is fed into the DDM generator along with the stream of I/Q samples of the (up to) four beams that point to the specular reflection points over the sea surface of the reflected GPS signals with more strength. Every clock cycle a new pair of I/Q samples are processed: for each Doppler frequency and delay coordinates a frequency $\left(f_{L 1}=1575.42 \mathrm{MHz}+f_{\text {Doppler }}\right)$ and a sample of the PRN code of the particular reflected GPS signal are obtained. Then, they are combined with the I/Q samples, and finally they are sent to their corresponding accumulator block. At the end of the integration period, related to the PRN chip rate and to the coherence time of the sea surface, the result is dumped, obtaining a complex DDM point. This GPS-reflectometer computes all these DDM points at the same time, so after the selected integration time a whole DDM is available.

The design has been verified and the digital description has been largely simplified by developing a whole software emulator (simulating noise samples) using Matlab. Simulation results have been satisfactorily compared to the theoretical predictions using only one analog receiver and one antenna. Fig. 5 shows a laboratory setup of the one-receiver version 


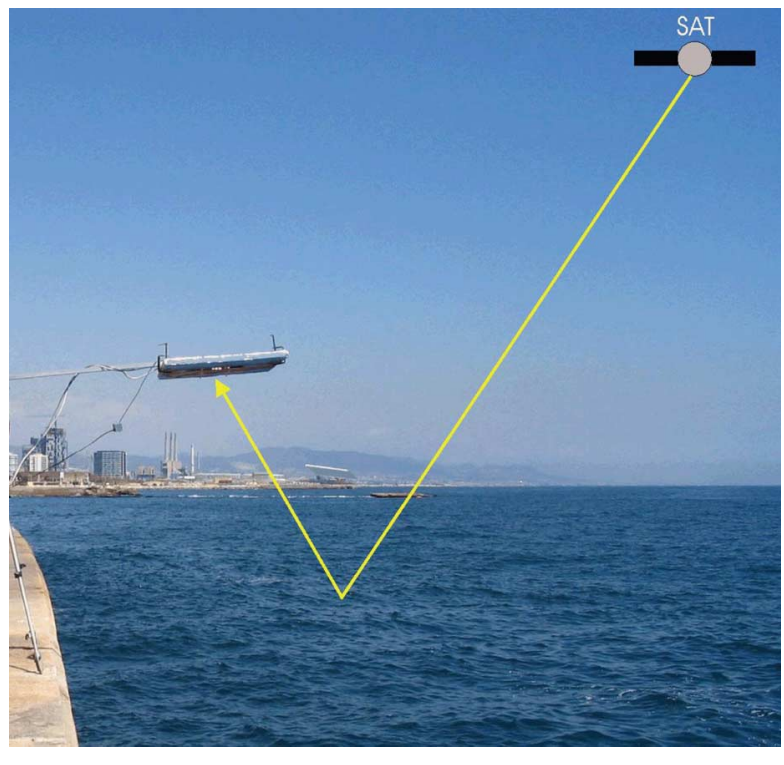

Fig. 6. Testing the system with one PAU receiver at the Barcelona Olympic Village harbor. The antenna is pointing to the sea surface to collect the reflected GPS signal and measure the brightness temperature (not in optimal conditions in this configuration). An auxiliary GPS receiver is used to collect the direct signal.

of the instrument. It includes the PAU receiver integrated behind the dual-polarization patch antenna, which is connected to the ADC board through a pair of Ethernet cables. The four sampled channels $\left(f_{s}=5.745 \mathrm{MHz}\right)$ of each receiver are timemultiplexed by a factor of 4 before they are input to the master FPGA. Master and slave FPGAs operate synchronously with the same clock and exchange information through a high-speed Mictor cable. The data processing includes Stokes parameters computation and DDM generation, and in the $4 \times 4$ array the generation of the different beams for both the radiometer (PAURAD) and the reflectometer (PAU-GNSS/R). The system is remotely controlled, and final products are sent to the external world through an Ethernet link. All receivers are driven by the same $10-\mathrm{MHz}$ reference clock and by a common noise source used for correlated noise injection.

Fig. 6 shows a test of the system with one PAU receiver at the Barcelona Olympic Village harbor in which the PAU antenna is pointing to the sea surface to collect the reflected GPS signal and measure the brightness temperature (although these are not the optimal conditions to measure the brightness temperature) and an auxiliary GPS receiver is used to collect the direct signal. Fig. 7 presents a sample DDM obtained with data acquired in a field experiment test in the Garraf cliffs, about $40 \mathrm{~km}$ south of Barcelona, on April 27, 2005.

\section{E. IR Radiometers}

Finally, the IR radiometers are two thermal IR commercial radiometers covering the spectral range $8-14 \mu \mathrm{m}$ that are connected to the serial ports of the control computer. One of them will be pointed at an incidence angle with respect to nadir of $30^{\circ}$ approximately, for which the influence of sea state is negligible [31]. The second one is used to measure the downwelling radiation at $60^{\circ}-70^{\circ}$ from zenith (so as to

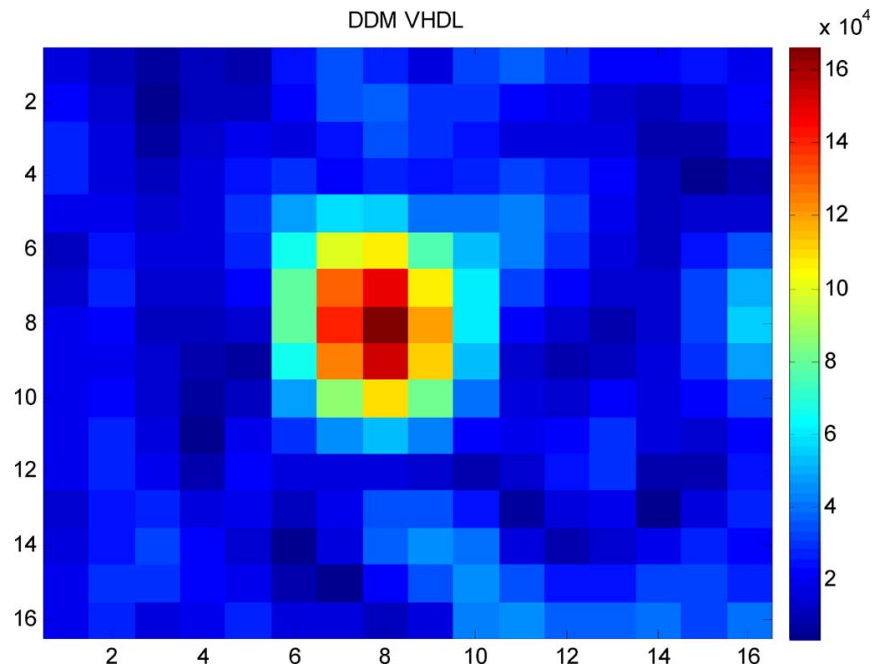

Fig. 7. Sample DDM obtained applying the VHDL generator. Data acquired for PRN19, at the Garraf cliffs, South of Barcelona, April 27, 2005.

have better radiometric sensitivity) and, in conjunction with MODTRAN simulations, is used to correct for atmospheric effects (mainly clouds and rain) [32].

\section{PAU-Radiometer Analysis Using Noise Waves}

In order to understand the operation of the PAU-RAD concept, the analysis of a single element is first performed using noise waves. Then, results are extended to the general case of an array of $N$ elements and performing DBF.

\section{A. Analysis of a Single-Element PAU-Radiometer}

The analysis of the single-element PAU-RAD using noise waves $^{3}$ is described in Fig. 8 and detailed in Appendix I. The final result is repeated here for convenience. The crosscorrelation matrix of the outgoing noise waves of the power splitter plus the equivalent noise waves at the amplifiers' input is given by

$$
\begin{aligned}
\mathbf{N}=\left\langle\mathbf{b}^{\prime} \mathbf{b}^{\prime \mathbf{H}}\right\rangle & =k_{\mathrm{B}} \cdot\left\{\boldsymbol{\Lambda} \cdot \mathbf{S} \cdot\left[\begin{array}{cccc}
T_{\mathrm{a}} \eta+T_{\mathrm{ph}}(1-\eta) & 0 & 0 & \\
0 & T_{\mathrm{r} 1} & 0 \\
0 & 0 & T_{\mathrm{r} 2}
\end{array}\right] \cdot \mathbf{S}^{\mathbf{H}} \cdot \boldsymbol{\Lambda}^{\mathbf{H}}\right. \\
& +\left[\begin{array}{ccc}
0 & 0 & 0 \\
0 & T_{\mathrm{R} 1} & 0 \\
0 & 0 & T_{\mathrm{R} 2}
\end{array}\right]+\boldsymbol{\Lambda} \cdot T_{\mathrm{ph}}\left(\mathbf{I}-\mathbf{S} \cdot \mathbf{S}^{\mathbf{H}}\right) \cdot \mathbf{\Lambda}^{\mathbf{H}} \\
& \left.+\boldsymbol{\Lambda} \cdot \mathbf{S} \cdot\left[\begin{array}{ccc}
0 & 0 & 0 \\
0 & T_{\mathrm{c} 1} & 0 \\
0 & 0 & T_{\mathrm{c} 2}
\end{array}\right]+\left[\begin{array}{ccc}
0 & 0 & 0 \\
0 & T_{\mathrm{c} 1}^{*} & 0 \\
0 & 0 & T_{\mathrm{c} 2}^{*}
\end{array}\right] \cdot \mathbf{S}^{\mathbf{H}} \cdot \boldsymbol{\Lambda}^{\mathbf{H}}\right\} .
\end{aligned}
$$

\footnotetext{
${ }^{3}$ The analysis is performed assuming constant complex noise waves over a $1-\mathrm{Hz}$ bandwidth. Over the radiometer's bandwidth, the complex noise waves can be different, and the final result is obtained by integrating the responses over the total bandwidth
} 


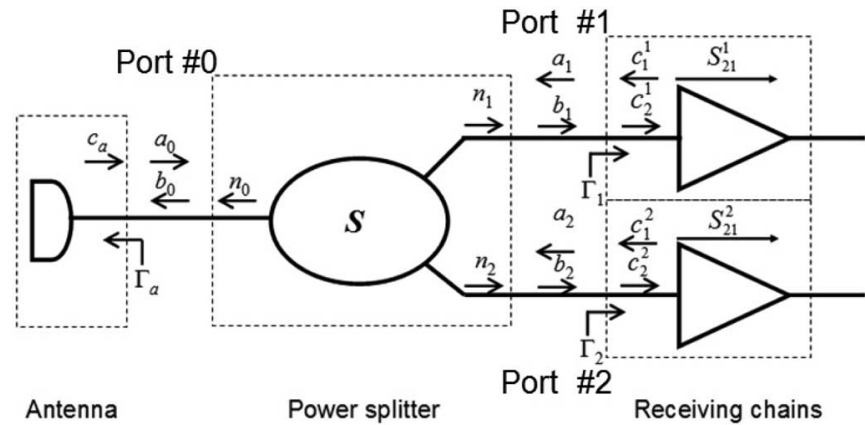

Fig. 8. Noise wave analysis of the PAU-RAD. Wilkinson power splitter input is labeled as \#0, and its outputs are labeled as \#1 and \#2.

The first term in (1) contains the radiometer's output correlation from the input noise (the one collected by the antenna and generated by the antenna losses). The second term contains the correlation of the noise that is generated by each receiver and appears at their outputs. Since these noise waves are uncorrelated among the different amplifiers, the second matrix is diagonal. The third term contains the correlation of the noise generated by the power divider network. Note that this term would vanish if this network where unitary $\left(\mathbf{I}=\mathbf{S} \cdot \mathbf{S}^{\mathbf{H}}\right)$, which is not the case for a Wilkinson power divider since ideally $S_{12}=S_{21}=0$. Finally, the fourth and fifth ones contain the radiometers' output generated by the outgoing noise that enters in the power divider and leaks into the other output port due to the finite isolation.

The radiometer's output is the cross correlation of the outputs at ports 1 and $2\left(N^{1,2}\right)$ passed through the receiving chains $\left(V^{1,2}\right)$, and it can be computed as

$$
V^{1,2}=S_{21}^{1} \cdot S_{21}^{2 *} \cdot N^{1,2}
$$

where $S_{21}^{i}$ is the $S_{21}$ parameter of the $i$ th receiving channel of the element.

In the general case, the expansion of (1) and (2) is quite cumbersome, and even though (2) can be developed analytically, the physical meaning is lost. In order to gain insight in the process, several particular cases of interest are studied below.

1) Perfect matching of the antenna and receivers' input ports $\Gamma_{a}=\Gamma_{1}=\Gamma_{2}=0$ :

$$
\begin{aligned}
V^{1,2}=S_{21}^{1} \cdot S_{21}^{2 *} \cdot N^{1,2} & \\
=S_{21}^{1} \cdot S_{21}^{2 *} \cdot k_{\mathrm{B}}\{ & S_{10} \cdot S_{20}^{*}\left[T_{\mathrm{a}} \eta+T_{\mathrm{ph}}(1-\eta)\right] \\
& +S_{11} \cdot S_{21}^{*} \cdot T_{\mathrm{r} 1}+S_{12} \cdot S_{22}^{*} \cdot T_{\mathrm{r} 2} \\
& +S_{12} \cdot T_{\mathrm{c} 2}+S_{21}^{*} \cdot T_{\mathrm{c} 1}-T_{\mathrm{ph}} \\
& \left.\times\left(S_{10} \cdot S_{20}^{*}+S_{11} \cdot S_{21}^{*}+S_{12} \cdot S_{22}^{*}\right)\right\}
\end{aligned}
$$

where the second and third terms correspond to outputs originated by reflections of the outgoing noise waves from the receiving chains in the Wilkinson power divider output ports. The third and fourth terms correspond to correlations between the outgoing noise waves from the receiving chains that leak through the power divider and enter into the other chain.
2) Perfect matching of the antenna and receivers' input ports $\Gamma_{a}=\Gamma_{1}=\Gamma_{2}=0$, and of the Wilkinson power divider $S_{00}=S_{11}=S_{22}=0$ :

$$
\begin{aligned}
V^{1,2}= & S_{21}^{1} \cdot S_{21}^{2 *} \cdot N^{1,2} \\
=S_{21}^{1} \cdot S_{21}^{2 *} \cdot k_{\mathrm{B}} & {\left[S_{10} \cdot S_{20}^{*}\left(T_{\mathrm{a}}-T_{\mathrm{ph}}\right)\right.} \\
& \left.\quad+S_{12} \cdot T_{\mathrm{c} 2}+S_{21}^{*} \cdot T_{\mathrm{c} 1}\right] .
\end{aligned}
$$

3) Perfect matching of the antenna and receivers' input ports $\Gamma_{a}=\Gamma_{1}=\Gamma_{2}=0$, and of the Wilkinson power divider $S_{00}=S_{11}=S_{22}=0$, and perfect isolation of its outputs $S_{12}=S_{21}=0$ :

$$
\begin{aligned}
V^{1,2} & =S_{21}^{1} \cdot S_{21}^{2 *} \cdot N^{1,2} \\
& =S_{21}^{1} \cdot S_{21}^{2 *} \cdot S_{10} \cdot S_{20}^{*} \cdot k_{\mathrm{B}}\left(T_{\mathrm{a}}-T_{\mathrm{ph}}\right) .
\end{aligned}
$$

Note the following.

a) The radiometer's output is proportional to the difference between the antenna temperature, and the physical temperature of the antenna and Wilkinson power divider, as in a Dicke radiometer, but without the input switch.

b) If not calibrated, (3)-(5) contain a term which is proportional to $T_{\mathrm{a}}-T_{\mathrm{ph}}$ and an offset term that depends on the receiving chains noise temperatures. This offset can be canceled by injection of uncorrelated noise (Fig. 2) and by subtracting the two measured cross correlations when the input switches are connected to the antenna and to the matched loads.

c) If not calibrated, (5) can be a complex number due to imperfect mismatch of the two receiving chains and of the Wilkinson power divider. This error is corrected by the injection of correlated noise (Fig. 2) and by compensating the phase of the measured cross correlation (5) when the input switch is connected to the antenna, with the phase of the measured cross correlation (5) when the input switches are connected to the correlated noise source (Fig. 2).

d) As compared to the differential microwave radiometer [33], both receiving chains do not have to be perfectly matched in amplitude and phase, which significantly relaxes the instrument specifications.

\section{B. Analysis of the PAU-Radiometer Formed by an Array of Elements With $D B F$}

As discussed in Section II, PAU-RAD consists of an array of $4 \times 4$ dual-polarization receiving elements, with two channels per polarization each. That is, the total number of channels is 64, whose signals are sampled at eight bits and calibrated in phase and amplitude using uncorrelated/correlated noise injection in a similar way as it is done in SMOS [34]. Then, at each polarization $p$, the calibrated signals are added with appropriate complex weights $\left(\beta_{n}\right)$ so as to form beams in different directions, avoiding the need to mechanically scan the antenna. 


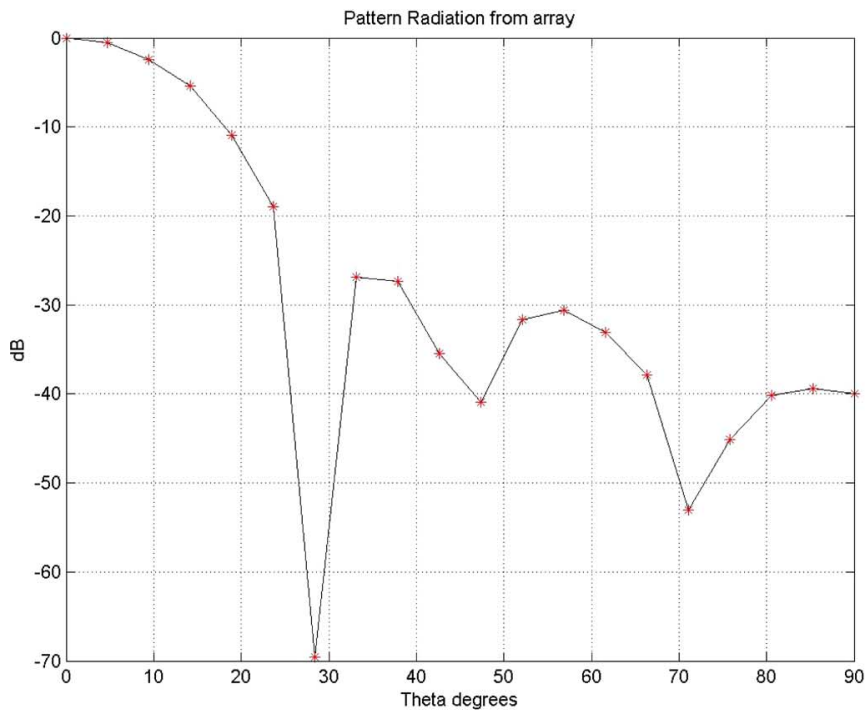

(a)

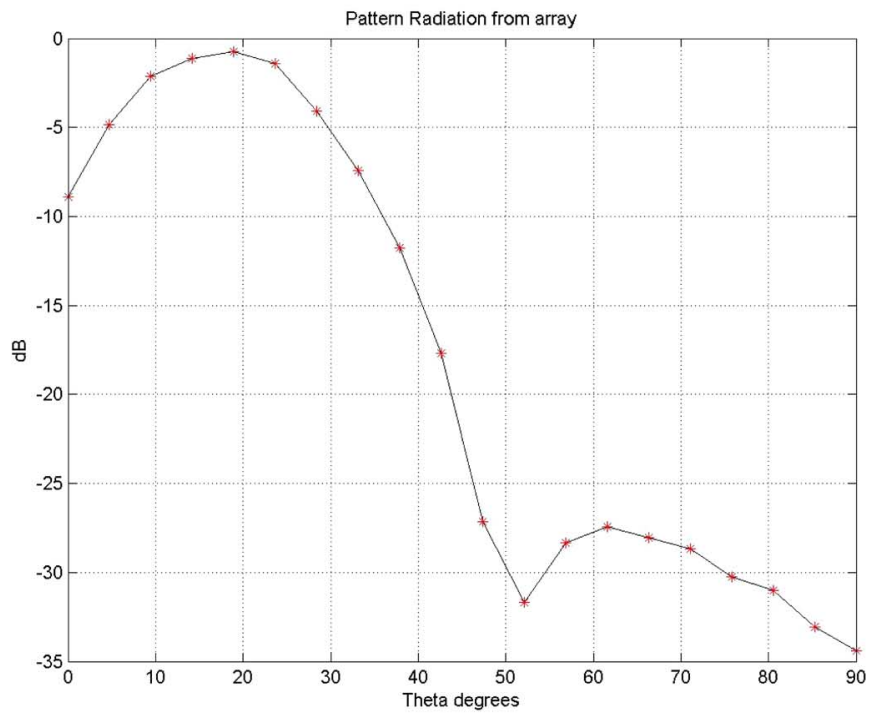

(b)

Fig. 9. Simulated synthetic beam at (a) the boresight direction and (b) $20^{\circ}$ off boresight, including the radiation pattern of the elementary element.

Following the same nomenclature as in Appendix I, the output signals of the DBF are computed as follows:

$$
\begin{aligned}
& S_{p+}=\sum_{n=1}^{N} \beta_{n}\left(\frac{1}{\sqrt{2}}\left(c_{a, p}^{n}+n_{1, p}^{n}\right)+c_{2, p}^{1, n}\right) \\
& S_{p-}=\sum_{m=1}^{N} \beta_{m}\left(\frac{1}{\sqrt{2}}\left(c_{a, p}^{m}+n_{2, p}^{m}\right)+c_{2, p}^{2, m}\right)
\end{aligned}
$$

where:

1) $S_{p+}$ and $S_{p-}$ are the complex DBF outputs at $p$ polarization $(p=\nu, h$ : vertical or horizontal): The + subscript corresponds to the receiving chain where the antenna signal and the noise signal generated by the Wilkinson power splitter resistor are added in phase (by definition, the upper receiving chain, see Fig. 8), and the - subscript corresponds to the branch where they are added $180^{\circ}$ out of phase (by definition, the lower receiving chain, see Fig. 8).

2) $\beta_{i}$ is the complex weight for each array element.

3) $c_{a, p}^{n}$ is the signal at the output of the $p$-polarization antenna of the $n$th element (Fig. 8).

4) $n_{1, p}^{n}$ and $n_{2, p}^{n}$ are the noise signals generated by the Wilkinson power splitter of the $n$th element at $p$ polarization $\left(n_{1, p}^{n}=-n_{2, p}^{n} \triangleq n_{p}^{n}\right)$.

5) $c_{1, p}^{1, n}$ and $c_{1, p}^{2, n}$ are the equivalent noise waves at the amplifiers' input (Fig. 8).

The first and second Stokes parameters are obtained from the cross correlation of the signals in (6) at $\nu$ - and $h$-polarizations

$$
\begin{aligned}
& T_{1}=\Re e\left\langle S_{\nu+} \cdot S_{\nu-}^{*}\right\rangle \\
& T_{2}=\Re e\left\langle S_{h+} \cdot S_{h-}^{*}\right\rangle
\end{aligned}
$$

which after some manipulations (see Appendix II) can be expressed as

$$
\begin{aligned}
& T_{1}=T_{a, \nu}-T_{\mathrm{ph}} \\
& T_{2}=T_{a, h}-T_{\mathrm{ph}} .
\end{aligned}
$$

The third and fourth Stokes parameters $\left(T_{3}\right.$ and $T_{4}$, or $\mathrm{U}$ and V) are computed by cross-correlating the signals at different polarizations $S_{h+}$ and $S_{\nu-}$ (see Appendix II)

$$
\begin{aligned}
& T_{3}=2 \cdot \Re e\left[\left\langle S_{h+} \cdot S_{\nu-}^{*}\right\rangle\right] \hat{=} 2 \cdot \Re e\left[T_{a, h \nu}\right] \\
& T_{4}=2 \cdot \Im m\left[\left\langle S_{h+} \cdot S_{\nu-}^{*}\right\rangle\right] \hat{=} 2 \cdot \Im m\left[T_{a, h \nu}\right] .
\end{aligned}
$$

The use of the third and fourth Stokes parameter can be used to correct for rotations of the antenna reference frame with respect to the earth's reference frame and/or to correct Faraday rotation for a spaceborne instrument as described in [36, eq. (10)]:

$$
\lambda_{1,2}=\frac{T_{1}+T_{2}}{2} \pm \frac{\sqrt{\left(T_{1}-T_{2}\right)^{2}+\left|T_{3}+j T_{4}\right|^{2}}}{2}
$$

where the estimated $T_{1}$ and $T_{2}$ are $\lambda_{1}$ and $\lambda_{2}$, respectively, and in deriving (10) from [36, eq. (10)], we have made use of $T_{\mathrm{VH}}=\left(T_{3}+j T_{4}\right) / 2$.

\section{PAU-RAdiometer Performance}

\section{A. Angular Resolution}

The angular resolution of this type of radiometer is the same as that of an array of $\sqrt{N} \times \sqrt{N}$ elements, spaced $d$ wavelengths in both dimensions and with a given illumination window.

To analyze the performance of PAU-RAD, a Matlab-based instrument simulator has been developed. It generates bandpass noise samples that are then propagated to the different antenna elements, passed through the receiving chains, calibrated, and finally combined so as to synthesize different antenna beams. In our case: $N=16$ (square array of $4 \times 4$ elements), $d=0.63$ wavelengths, and the amplitude of the weighting 
function is the product of two triangular ones [lllllll $\left.\begin{array}{lll}2 & 2 & 1\end{array}\right]$ in both dimensions:

$$
|\beta|=\frac{1}{10}\left[\begin{array}{cccc}
1 & 2 & 2 & 1 \\
2 & 4 & 4 & 2 \\
2 & 4 & 4 & 2 \\
1 & 2 & 2 & 1
\end{array}\right]
$$

normalized so that the sum of the squares of all elements equals 1 (A2.4). A linear phase shift is applied to each row in (10) to steer the beam out of the boresight direction.

Fig. 9 shows the simulated results when the synthesized beam is pointed toward the boresight direction [Fig. 9(a)] and $20^{\circ}$ out-of-boresight [Fig. 9(b)]. The number of points in the simulated beam is however limited due to the long simulation times. The elementary radiation pattern of each array element (a microstrip patch antenna) is included in the simulations, which explains the decrease in the maximum's amplitude when the beam is pointed out-of-boresight. The level of the secondary lobes is, as expected, about $26 \mathrm{~dB}$, and the MBE larger than $95 \%$ even at $20^{\circ}$ out-of-boresight.

\section{B. Radiometric Sensitivity}

Assuming that all receiving elements are at the same physical temperature $\left(T_{\mathrm{ph}}\right)$ and that all the receivers noise temperature $\left(T_{R}\right)$ are the same, the radiometric sensitivity of the Stokes parameters can be readily computed after some lengthy manipulations (see Appendix III) as

$$
\begin{aligned}
\Delta T_{1} & =\frac{T_{a, \nu}+T_{\mathrm{R}}+2 \cdot T_{\mathrm{ph}}}{\sqrt{B \cdot \tau}} \\
\Delta T_{2} & =\frac{T_{a, h}+T_{\mathrm{R}}+2 \cdot T_{\mathrm{ph}}}{\sqrt{B \cdot \tau}}
\end{aligned}
$$

and

$$
\Delta T_{3,4}=\sqrt{2} \cdot \frac{\sqrt{\left(T_{a, h}+T_{\mathrm{R}}+2 \cdot T_{\mathrm{ph}}\right) \cdot\left(T_{a, \nu}+T_{\mathrm{R}}+2 \cdot T_{\mathrm{ph}}\right)}}{\sqrt{B \cdot \tau}}
$$

where $B$ is the predetection bandwidth and $\tau$ is the integration time.

It is interesting to note that the radiometric sensitivity of the first two Stokes parameters corresponds to that of a total power radiometer in which the system temperature is increased by $2 \cdot T_{\mathrm{ph}}$ due to the noise added by the resistor of the Wilkinson power splitter.

The result for the radiometric sensitivity of the third and fourth Stokes parameters is also intuitive, with the replacement of the system temperatures in both channels by new ones, including also the term $+2 \cdot T_{\mathrm{ph}}$.

\section{CONCLUSION}

This paper has summarized the main objectives, and has presented the topology and the main characteristics of the PAU instrument. PAU consists of three different instruments: an L-band radiometer to measure the sea surface brightness temperature, a GPS-reflectometer to infer the sea state from the DDMs, and a pair of IR radiometers to measure the sea surface temperature, being the sea-state information and the sea surface temperature necessary variables in order to retrieve the sea surface salinity.

The key characteristic of PAU is that both the radiometer and the GPS-reflectometer operate simultaneously (there is no time multiplex) and share completely the RF, IF, and ADC sections. To achieve this goal, a new receiver topology has been devised, which consists of two receiving chains and a cross-correlator. This topology can be applied to both a single antenna and radiometer receiver, or to an array of antennas and receiving elements using DBF. The analysis of this new type of radiometer has been performed using noise waves and it has been shown that its output is the same as for a Dicke radiometer: the difference between the antenna temperature and the physical temperature of the Wilkinson power splitter. This radiometer topology does not require amplitude and phase matching between the two receiving chains, since calibration using correlated/uncorrelated noise injection can correct for them. It can also be applied to radioastronomy observations as well if the front-end is cooled to reduce the physical temperature of the Wilkinson power splitter. Finally, the radiometer performance has been analyzed in terms of the angular resolution and the radiometric sensitivity.

A ground-based and a UAV-based versions of PAU have already been developed and the full $4 \times 4$ array is currently under integration

\section{APPENDIX I \\ Single-ElEment PAU-RAD ANALYSis USING NOISE W AVES}

A linear $N$-port can be represented by its $N \times N$ scattering matrix (S) and by a $N \times 1$ noise vector $(\mathbf{n})$ that accounts for the noise waves generated internally [25]

$$
\mathbf{b}=\mathbf{S} \cdot \mathbf{a}+\mathbf{n}
$$

where $\mathbf{a}$ and $\mathbf{b}$ are the usual incident and outgoing $N \times 1$ wave vectors defined over a $1-\mathrm{Hz}$ bandwidth. The incident wave vector can also be written as [26]

$$
\mathbf{a}=\boldsymbol{\Gamma} \cdot \mathbf{b}+\mathbf{a}_{\mathbf{s}}
$$

where $\mathbf{a}_{\mathbf{s}}$ is the vector of source waves connected to each of the $N$ ports. In our case (Fig. 9)

$$
\mathbf{a}_{\mathbf{s}}=\left[\begin{array}{c}
c_{a} \\
c_{1}^{1} \\
c_{1}^{2}
\end{array}\right]
$$

whose elements are $c_{a}$, which is the noise collected by the antenna from the outside, and the one generated internally because of its ohmic losses, and $c_{1}^{i}$ which is the noise generated by the $i$ th receiving chains toward the input.

On the other hand, $\boldsymbol{\Gamma}$ is a $N \times N$ diagonal matrix whose elements are the reflection coefficients of each of the ports as 
seen from the network (Fig. 8, the antenna and the two receiving chains)

$$
\boldsymbol{\Gamma}=\left[\begin{array}{ccc}
\Gamma_{a} & 0 & 0 \\
0 & \Gamma_{1} & 0 \\
0 & 0 & \Gamma_{2}
\end{array}\right]
$$

Substituting (A1.2) in (A1.1) and isolating $\mathbf{b}$, the following expression is readily obtained:

$$
\mathbf{b}=(\mathbf{I}-\mathbf{S} \cdot \boldsymbol{\Gamma})^{-1} \cdot \mathbf{S} \cdot \mathbf{a}_{\mathbf{s}}+(\mathbf{I}-\mathbf{S} \cdot \boldsymbol{\Gamma})^{-1} \cdot \mathbf{n}
$$

and defining

$$
\mathbf{\Lambda} \hat{=}(\mathbf{I}-\mathbf{S} \cdot \boldsymbol{\Gamma})^{-1}
$$

as in [27], (A1.5) can be rewritten in a more compact notation as

$$
\mathbf{b}=\boldsymbol{\Lambda} \cdot \mathbf{S} \cdot \mathbf{a}_{\mathbf{s}}+\boldsymbol{\Lambda} \cdot \mathbf{n} .
$$

Finally, to take into account the equivalent noise waves $c_{2}^{i}$ (Fig. 9) at the amplifiers' input, the $\mathbf{b}^{\prime}$ vector is defined

$$
\mathbf{b}^{\prime}=\mathbf{b}+\mathbf{c}
$$

in which $\mathbf{c}$ is defined as

$$
\mathbf{c}=\left[\begin{array}{c}
0 \\
c_{2}^{1} \\
c_{2}^{2}
\end{array}\right] .
$$

The radiometer's output is the cross correlation of the noise waves at the receivers' output

$$
V^{1,2}=S_{21}^{1} \cdot S_{21}^{2 *} \cdot\left\langle b_{1}^{\prime} \cdot b_{2}^{\prime *}\right\rangle
$$

where $S_{21}^{i}$ is the $S_{21}$ parameter of the $i$ th receiving chain. In order to compute (A1.10), we must first compute the correlation matrix $\mathbf{N}$ of vector $\mathbf{b}^{\prime}$

$$
\begin{aligned}
\mathbf{N}= & \left\langle\mathbf{b}^{\prime} \cdot \mathbf{b}^{\prime \mathbf{H}}\right\rangle \\
= & \left\langle\left(\boldsymbol{\Lambda} \cdot \mathbf{S} \cdot \mathbf{a}_{\mathbf{s}}+\boldsymbol{\Lambda} \cdot \mathbf{n}+\mathbf{c}\right) \cdot\left(\boldsymbol{\Lambda} \cdot \mathbf{S} \cdot \mathbf{a}_{\mathbf{s}}+\boldsymbol{\Lambda} \cdot \mathbf{n}+\mathbf{c}\right)^{\mathbf{H}}\right\rangle \\
= & \boldsymbol{\Lambda} \cdot \mathbf{S} \cdot\left\langle\mathbf{a}_{\mathbf{s}} \cdot \mathbf{a}_{\mathbf{s}}^{\mathbf{H}}\right\rangle \cdot \mathbf{S}^{\mathbf{H}} \cdot \boldsymbol{\Lambda}^{\mathbf{H}}+\boldsymbol{\Lambda} \cdot \mathbf{S} \cdot\left\langle\mathbf{a}_{\mathbf{s}} \cdot \mathbf{c}^{\mathbf{H}}\right\rangle \\
& +\boldsymbol{\Lambda} \cdot\left\langle\mathbf{n} \cdot \mathbf{n}^{\mathbf{H}}\right\rangle \cdot \boldsymbol{\Lambda}^{\mathbf{H}}+\left\langle\mathbf{c} \cdot \mathbf{a}_{\mathbf{s}}^{\mathbf{H}}\right\rangle \cdot \mathbf{S}^{\mathbf{H}} \cdot \boldsymbol{\Lambda}^{\mathbf{H}}+\left\langle\mathbf{c} \cdot \mathbf{c}^{\mathbf{H}}\right\rangle .
\end{aligned}
$$

(A1.11)

In evaluating (A1.11), we must first compute the correlation matrices of the different signals involved. The noise matrix of the incoming noise waves is a diagonal one, since the noise is coming from the antenna, and the noise waves generated by the receivers toward the input are uncorrelated

$$
\left\langle\mathbf{a}_{\mathbf{s}} \cdot \mathbf{a}_{\mathbf{s}}^{\mathbf{H}}\right\rangle=k_{\mathrm{B}}\left[\begin{array}{ccc}
T_{\mathrm{a}} \eta+T_{\mathrm{ph}}(1-\eta) & 0 & 0 \\
0 & T_{\mathrm{r} 1} & 0 \\
0 & 0 & T_{\mathrm{r} 2}
\end{array}\right] .
$$

And in a similar way we have

$$
\begin{aligned}
&\left\langle\mathbf{a}_{\mathbf{s}} \cdot \mathbf{c}^{\mathbf{H}}\right\rangle= {\left[\begin{array}{c}
c_{a} \\
c_{1}^{1} \\
c_{1}^{2}
\end{array}\right] \cdot\left[\begin{array}{lll}
0 & c_{2}^{1 *} & c_{2}^{2 *}
\end{array}\right]=k_{\mathrm{B}}\left[\begin{array}{ccc}
0 & 0 & 0 \\
0 & T_{\mathrm{c} 1} & 0 \\
0 & 0 & T_{\mathrm{c} 2}
\end{array}\right] } \\
&\left\langle\mathbf{c} \cdot \mathbf{a}_{\mathbf{s}}^{\mathbf{H}}\right\rangle=\left[\begin{array}{c}
0 \\
c_{2}^{1} \\
c_{2}^{2}
\end{array}\right] \cdot\left[\begin{array}{lll}
c_{a}^{*} & c_{1}^{1 *} & c_{1}^{2 *}
\end{array}\right]=k_{\mathrm{B}}\left[\begin{array}{ccc}
0 & 0 & 0 \\
0 & T_{\mathrm{c} 1}^{*} & 0 \\
0 & 0 & T_{\mathrm{c} 2}^{*}
\end{array}\right] \\
&\left\langle\mathbf{c} \cdot \mathbf{c}^{\mathbf{H}}\right\rangle=\left[\begin{array}{c}
0 \\
c_{2}^{1} \\
c_{2}^{2}
\end{array}\right] \cdot\left[\begin{array}{lll}
0 & c_{2}^{1 *} & c_{2}^{2 *}
\end{array}\right]=k_{\mathrm{B}}\left[\begin{array}{ccc}
0 & 0 & 0 \\
0 & T_{\mathrm{R} 1} & 0 \\
0 & 0 & T_{\mathrm{R} 2}
\end{array}\right]
\end{aligned}
$$

and [25]

$$
\left\langle\mathbf{n} \cdot \mathbf{n}^{\mathbf{H}}\right\rangle=k_{\mathrm{B}} \cdot T_{\mathrm{ph}}\left(\mathbf{I}-\mathbf{S} \cdot \mathbf{S}^{\mathbf{H}}\right) .
$$

In the previous equations, $k_{\mathrm{B}}$ is the Boltmann's constant, $T_{\mathrm{a}}$ is the antenna temperature at a given polarization, $\eta$ is the antenna ohmic losses, $T_{\mathrm{ph}}$ is the physical temperature of the antenna and of the Wilkinson power splitter (assumed to be the same), $T_{\text {ri }}$, $T_{\mathrm{ci}}$, and $T_{\mathrm{Ri}}$ are the three receiver equivalent noise temperatures defined in [28], and $T_{\mathrm{Ri}}$ are the receivers' noise temperature with the standard definition for zero reflection coefficient [25].

Substituting (A1.12)-(A1.16) in (A1.11) we have

$$
\begin{aligned}
& \mathbf{N}=k_{\mathrm{B}} \cdot\left\{\boldsymbol{\Lambda} \cdot \mathbf{S} \cdot\left[\begin{array}{ccc}
T_{\mathrm{a}} \eta+T_{\mathrm{ph}}(1-\eta) & 0 & 0 \\
0 & T_{\mathrm{r} 1} & 0 \\
0 & 0 & T_{\mathrm{r} 2}
\end{array}\right] \cdot \mathbf{S}^{\mathbf{H}} \cdot \boldsymbol{\Lambda}^{\mathbf{H}}\right. \\
& +\left[\begin{array}{ccc}
0 & 0 & 0 \\
0 & T_{\mathrm{R} 1} & 0 \\
0 & 0 & T_{\mathrm{R} 2}
\end{array}\right]+\mathbf{\Lambda} \cdot T_{\mathrm{ph}}\left(\mathbf{I}-\mathbf{S} \cdot \mathbf{S}^{\mathbf{H}}\right) \cdot \mathbf{\Lambda}^{\mathbf{H}} \\
& \left.+\boldsymbol{\Lambda} \cdot \mathbf{S} \cdot\left[\begin{array}{ccc}
0 & 0 & 0 \\
0 & T_{\mathrm{c} 1} & 0 \\
0 & 0 & T_{\mathrm{c} 2}
\end{array}\right]+\left[\begin{array}{ccc}
0 & 0 & 0 \\
0 & T_{\mathrm{c} 1}^{*} & 0 \\
0 & 0 & T_{\mathrm{c} 2}^{*}
\end{array}\right] \cdot \mathbf{S}^{\mathbf{H}} \cdot \boldsymbol{\Lambda}^{\mathbf{H}}\right\}
\end{aligned}
$$

from which the radiometer's output can be computed as

$$
V^{1,2}=S_{21}^{1} \cdot S_{21}^{2 *} \cdot N^{1,2}
$$

where $S_{21}^{i}$ is the $S_{21}$ parameter of the $i$ th receiver, and $N^{1,2}$ is the element in the first row and second column of matrix $\mathbf{N}$, similarly as in [29], [30].

\section{APPENDIX II \\ COMPUTATION OF THE STOKES \\ PARAMETERS IN PAU-RAD}

In the following derivations, it is implicitly assumed that the signals are calibrated so that the radiometer's output has units of kelvin, and not watts. That is, the integration over a bandwidth $B$ is already performed and the results are divided by $k_{\mathrm{B}} \cdot B$. 
Derivation of the first and second Stokes parameters:

$$
\begin{aligned}
T_{1,2}= & \Re e\left(\left\langle S_{p+} \cdot S_{p-}^{*}\right\rangle\right) \\
= & \Re e\left(\left\langle\sum_{n=1}^{N} \beta_{n}\left(\frac{1}{\sqrt{2}}\left(c_{a, p}^{n}+n_{p}^{n}\right)+c_{2, p}^{1, n}\right)\right.\right. \\
& \left.\left.\cdot \sum_{m=1}^{N} \beta_{m}^{*}\left(\frac{1}{\sqrt{2}}\left(c_{a, p}^{m}-n_{p}^{m}\right)+c_{2, p}^{1, m}\right)^{*}\right\rangle\right) \\
= & \Re e\left(\left\langle\sum_{n=1}^{N} \sum_{m=1}^{N} \beta_{n} \cdot \beta_{m}^{*} \cdot\left(\frac{1}{\sqrt{2}}\left(c_{a, p}^{n}+n_{p}^{n}\right)+c_{2, p}^{1, n}\right)\right.\right. \\
& \left.\left.\cdot\left(\frac{1}{\sqrt{2}}\left(c_{a, p}^{m}-n_{p}^{m}\right)+c_{2, p}^{1, m}\right)^{*}\right\rangle\right) . \quad \text { (A2.1 }
\end{aligned}
$$

Taking the following into account.

1) For each element, the signal collected by the antenna, the noise generated by the Wilkinson power splitter and the receivers noise are uncorrelated.

2) The noise generated by the Wilkinson power splitter of each element is uncorrelated to the others.

3) The receiver's noise of each element is uncorrelated to the others, (A2.1) can be simplified as

$$
\begin{aligned}
T_{1,2}= & \Re e\left(\left\langle S_{p+} \cdot S_{p-}^{*}\right\rangle\right) \\
= & \Re e\left(\frac{1}{2} \sum_{n=1}^{N} \sum_{m=1}^{N} \beta_{n} \cdot \beta_{m}^{*} \cdot\left\langle c_{a, p}^{n} \cdot c_{a, p}^{m *}\right\rangle\right. \\
& \left.\quad-\frac{1}{2} \sum_{n=1}^{N}\left|\beta_{n}\right|^{2} \cdot\left\langle\left|n_{p}^{n}\right|^{2}\right\rangle\right) .
\end{aligned}
$$

Since the antenna temperature, as measured by the array, is equal to

$$
\begin{aligned}
T_{a, p} & \triangleq\left\langle\sum_{n=1}^{N} \frac{1}{\sqrt{2}} \beta_{n} \cdot c_{a, p}^{n} \cdot \sum_{m=1}^{N} \frac{1}{\sqrt{2}} \beta_{m}^{*} \cdot c_{a, p}^{m *}\right\rangle \\
& \equiv \sum_{n=1}^{N} \sum_{m=1}^{N} \frac{1}{2} \cdot \beta_{n} \cdot \beta_{m}^{*} \cdot\left\langle c_{a, p}^{n} \cdot c_{a, p}^{m *}\right\rangle
\end{aligned}
$$

and

$$
\sum_{n=1}^{N}\left|\beta_{n}\right|^{2} \triangleq 1
$$

the results for the first two Stokes parameters are, as in a Dicke radiometer, the difference between the antenna temperature at a given polarization minus the reference temperature

$$
\begin{aligned}
& T_{1}=T_{a, \nu}-T_{\mathrm{ph}} \\
& T_{2}=T_{a, h}-T_{\mathrm{ph}} .
\end{aligned}
$$

The derivation of the third and fourth Stokes parameters is similar

$$
\begin{aligned}
& T_{3}=2 \cdot \Re e\left[\left\langle S_{h+} \cdot S_{\nu-}^{*}\right\rangle\right] \\
& T_{4}=2 \cdot \Im m\left[\left\langle S_{h+} \cdot S_{\nu-}^{*}\right\rangle\right] .
\end{aligned}
$$

Expanding the product within brackets we have

$$
\begin{array}{r}
\left\langle S_{h+} \cdot S_{\nu-}^{*}\right\rangle=\left(\left\langle\sum_{n=1}^{N} \beta_{n}\left(\frac{1}{\sqrt{2}}\left(c_{a, h}^{n}+n_{h}^{n}\right)+c_{2, h}^{1, n}\right)\right.\right. \\
\left.\left.\cdot \sum_{m=1}^{N} \beta_{m}^{*}\left(\frac{1}{\sqrt{2}}\left(c_{a, \nu}^{m}-n_{\nu}^{m}\right)+c_{2, \nu}^{1, m}\right)^{*}\right\rangle\right) \\
=\sum_{n=1}^{N} \sum_{m=1}^{N}\left[\beta_{n} \cdot \beta_{m}^{*} \cdot\left(\frac{1}{\sqrt{2}}\left(c_{a, h}^{n}+n_{h}^{n}\right)+c_{2, h}^{1, n}\right)\right. \\
\left.\cdot\left(\frac{1}{\sqrt{2}}\left(c_{a, \nu}^{m}-n_{\nu}^{m}\right)+c_{2, \nu}^{1, m}\right)^{*}\right]
\end{array}
$$

and, taking into account the previous considerations about the uncorrelation between the antenna signals, the noise generated by the Wilkinson power splitter and the receivers noise at each element; and the uncorrelation among the signals generated by different Wilkinson power splitters; or among the receivers noise of different elements, then

$\left\langle S_{h+} \cdot S_{\nu-}^{*}\right\rangle=\frac{1}{2} \sum_{n=1}^{N} \sum_{m=1}^{N} \beta_{n} \cdot \beta_{m}^{*} \cdot\left\langle c_{a, h}^{n} \cdot c_{a, \nu}^{m *}\right\rangle \triangleq T_{a, h \nu}$

and

$$
\begin{aligned}
& T_{3}=2 \cdot \Re e\left[T_{a, h \nu}\right] \\
& T_{4}=2 \cdot \Im m\left[T_{a, h \nu}\right] .
\end{aligned}
$$

\section{APPENDIX III}

\section{PAU-RAD RADIOMETRIC SENSITIVITY}

As in Appendix II, the signals are calibrated and the radiometer's output has units of kelvin. Assuming that all receiving elements are at the same physical temperature $\left(T_{\mathrm{ph}}\right)$ and that all the receivers noise temperature $\left(T_{R}\right)$ are the same, the radiometric sensitivity of the first and second Stokes parameters can be computed as $(p=h, \nu)$ from the standard deviation of the radiometer's output

$$
\begin{aligned}
\sigma^{2} & =\left\langle\left(S_{p+} \cdot S_{p-}^{*}\right) \cdot\left(S_{p+} \cdot S_{p-}^{*}\right)^{*}\right\rangle-\left\langle S_{p+} \cdot S_{p-}^{*}\right\rangle^{2} \\
& =\left\langle S_{p+} \cdot S_{p-}^{*} \cdot S_{p+}^{*} \cdot S_{p-}\right\rangle-\left\langle S_{p+} \cdot S_{p-}^{*}\right\rangle^{2} .
\end{aligned}
$$

From the complex Gaussian moment theorem [36]

$\left\langle u_{1}^{*} \cdot u_{2}^{*} \cdot u_{3} \cdot u_{4}\right\rangle=\left\langle u_{1}^{*} \cdot u_{3}\right\rangle \cdot\left\langle u_{2}^{*} \cdot u_{4}\right\rangle+\left\langle u_{1}^{*} \cdot u_{4}\right\rangle \cdot\left\langle u_{2}^{*} \cdot u_{3}\right\rangle$

the fourth-order moment in (A3.1) can be decomposed into second-order moments

$$
\begin{aligned}
& \left\langle S_{p+} \cdot S_{p-}^{*} \cdot S_{p+}^{*} \cdot S_{p-}\right\rangle \\
& \quad=\left\langle S_{p+} \cdot S_{p-} \cdot S_{p+}^{*} \cdot S_{p-}^{*}\right\rangle \\
& =\underbrace{\left\langle S_{p+} \cdot S_{p+}^{*}\right\rangle}_{1 \mathrm{st} \text { term }} \cdot \underbrace{\left\langle S_{p-} \cdot S_{p-}^{*}\right\rangle}_{\text {2nd term }}+\underbrace{\left\langle S_{p+} \cdot S_{p-}^{*}\right\rangle}_{\text {3rd term }} \cdot \underbrace{\left\langle S_{p-} \cdot S_{p+}^{*}\right\rangle}_{\text {4th term }} .
\end{aligned}
$$


The first term in (A3.3) can be computed as

$$
\begin{aligned}
\left\langle S_{p+} \cdot S_{p+}^{*}\right\rangle= & \sum_{n=1}^{N} \sum_{m=1}^{N} \beta_{n} \cdot \beta_{m}^{*} \cdot\left(\frac{1}{\sqrt{2}}\left(c_{a, p}^{n}+n_{p}^{n}\right)+c_{2, p}^{1, n}\right) \\
& \cdot\left(\frac{1}{\sqrt{2}}\left(c_{a, p}^{m}+n_{p}^{m}\right)+c_{2, p}^{1, m}\right)^{*} \\
= & \frac{1}{2} \sum_{n=1}^{N} \sum_{m=1}^{N} \beta_{n} \cdot \beta_{m}^{*} \cdot\left\langle c_{a, p}^{n} \cdot c_{a, p}^{m *}\right\rangle \\
& +\frac{1}{2} \sum_{n=1}^{N} \sum_{m=1}^{N} \beta_{n} \cdot \beta_{m}^{*} \cdot\left\langle n_{p}^{n} \cdot n_{p}^{m *}\right\rangle \\
& +\sum_{n=1}^{N} \sum_{m=1}^{N} \beta_{n} \cdot \beta_{m}^{*} \cdot\left\langle c_{2, p}^{1, n} \cdot c_{2, p}^{1, m *}\right\rangle \\
= & \frac{1}{2} \sum_{n=1}^{N} \sum_{m=1}^{N} \beta_{n} \cdot \beta_{m}^{*} \cdot\left\langle c_{a, p}^{n} \cdot c_{a, p}^{m *}\right\rangle \\
& +\frac{1}{2} \sum_{n=1}^{N}\left|\beta_{n}\right|^{2} \cdot\left\langle c_{2, p}^{1, n} \cdot c_{2, p}^{1, n *}\right\rangle \\
& +\sum_{n=1}^{N}\left|\beta_{n}\right|^{2} \cdot\left\langle c_{2, p}^{1, n} \cdot c_{2, p}^{1, n *}\right\rangle \\
& +\mathrm{A} 3.4) \\
& \\
&
\end{aligned}
$$

where we have taken into account (A2.4), the uncorrelation between the antenna signals, the noise generated by the Wilkinson power splitter, and the receivers noise at each element; and the uncorrelation among the signals generated by different Wilkinson power splitters; or among the receivers noise of different elements.

In (A3.3), the second term can be computed as

$$
\left\langle S_{p-} \cdot S_{p-}^{*}\right\rangle=T_{a, p}+T_{\mathrm{R}}+2 \cdot T_{\mathrm{ph}}
$$

the third term as

$$
\left\langle S_{p+} \cdot S_{p-}^{*}\right\rangle=T_{1,2}
$$

and the fourth term as

$$
\left\langle S_{p-} \cdot S_{p+}^{*}\right\rangle=\left(\left\langle S_{p+} \cdot S_{p-}^{*}\right\rangle\right)^{*}=\left(T_{1,2}\right)^{*}=T_{1,2} .
$$

Finally, the second term in (A3.1) is equal to the square of (A3.6)

$$
\left\langle S_{p+} \cdot S_{p-}^{*}\right\rangle^{2}=T_{1,2}^{2}
$$

Substituting (A3.4) to (A3.8) in (A3.1), the variance of the output is computed

$$
\begin{aligned}
\sigma^{2} & =\left\langle S_{p+} \cdot S_{p-}^{*} \cdot S_{p+}^{*} \cdot S_{p-}\right\rangle-\left\langle S_{p+} \cdot S_{p-}^{*}\right\rangle^{2} \\
& =\left(T_{a, p}+T_{\mathrm{R}}+2 \cdot T_{\mathrm{ph}}\right)^{2}
\end{aligned}
$$

from which the radiometric sensitivity for a can be readily derived for an integration time $\tau$ as

$$
\begin{aligned}
\Delta T_{1} & =\frac{T_{a, \nu}+T_{\mathrm{R}}+2 \cdot T_{\mathrm{ph}}}{\sqrt{B \cdot \tau}} \\
\Delta T_{2} & =\frac{T_{a, h}+T_{\mathrm{R}}+2 \cdot T_{\mathrm{ph}}}{\sqrt{B \cdot \tau}}
\end{aligned}
$$

where $B$ is the predetection bandwidth (the bandwidth of the input signals, which corresponds to a correlation time $\tau_{c}=1 / B$ ) and $n \triangleq \tau / \tau_{c}=B \cdot \tau$ is the number of independent samples that are averaged [37, ch. 6], and therefore-assuming random Gaussian variables - the standard deviation reduces by a factor $\sqrt{n}$.

Similarly, the variance of the third and fourth Stokes parameters can be computed as

$$
\begin{aligned}
\sigma^{2}= & \left\langle\left(S_{h+} \cdot S_{\nu-}^{*}\right) \cdot\left(S_{h+} \cdot S_{\nu-}^{*}\right)^{*}\right\rangle-\left\langle S_{h+} \cdot S_{\nu-}^{*}\right\rangle \cdot\left\langle S_{h+} \cdot S_{\nu-}^{*}\right\rangle^{*} \\
= & \left\langle S_{h+} \cdot S_{\nu-}^{*} \cdot S_{h+}^{*} \cdot S_{\nu-}\right\rangle-\left|\left\langle S_{h+} \cdot S_{\nu-}^{*}\right\rangle\right|^{2} \\
= & \left\langle S_{h+} \cdot S_{h+}^{*}\right\rangle \cdot\left\langle S_{\nu-} \cdot S_{\nu-}^{*}\right\rangle+\left\langle S_{h+} \cdot S_{\nu-}^{*}\right\rangle \cdot \\
& \times\left\langle S_{\nu-} \cdot S_{h+}^{*}\right\rangle-\left\langle S_{h+} \cdot S_{\nu-}^{*}\right\rangle^{2} \\
\approx & \left\langle S_{h+} \cdot S_{h+}^{*}\right\rangle \cdot\left\langle S_{\nu-} \cdot S_{\nu-}^{*}\right\rangle
\end{aligned}
$$

where the first factor in (A3.12) is (A3.4) with $p=h$, and the second factor is (A3.5) with $p=\nu$.

$$
\begin{aligned}
\left\langle S_{h+} \cdot S_{h+}^{*}\right\rangle & =T_{a, h}+T_{\mathrm{R}}+2 \cdot T_{\mathrm{ph}} \\
\left\langle S_{\nu-} \cdot S_{\nu-}^{*}\right\rangle & =T_{a, \nu}+T_{\mathrm{R}}+2 \cdot T_{\mathrm{ph}}
\end{aligned}
$$

In deriving (A3.12), it has been assumed that $\left\langle S_{h+} \cdot S_{\nu-}^{*}\right\rangle$ is negligible in front of $\left\langle S_{h+} \cdot S_{h-}^{*}\right\rangle$ and $\left\langle S_{\nu+} \cdot S_{\nu-}^{*}\right\rangle$, which is a very good approximation [4, Fig. 2].

Inserting (A3.13) and (A3.14) in (A3.12), (A3.12) becomes

$\sigma^{2}=\left(T_{a, h}+T_{\mathrm{R}}+2 \cdot T_{\mathrm{ph}}\right) \cdot\left(T_{a, \nu}+T_{\mathrm{R}}+2 \cdot T_{\mathrm{ph}}\right)$

from which the radiometric sensitivity for a can be readily derived for an integration time $\tau$ as

$$
\Delta T_{a, h \nu}=\frac{\sqrt{\left(T_{a, h}+T_{\mathrm{R}}+2 \cdot T_{\mathrm{ph}}\right) \cdot\left(T_{a, \nu}+T_{\mathrm{R}}+2 \cdot T_{\mathrm{ph}}\right)}}{\sqrt{B \cdot \tau}}
$$

where $B$ and $B \cdot \tau$ are previously defined.

Since $T_{a, h \nu}$ is a complex random variable, and the variances of the real and imaginary parts are the same, their standard deviation is $1 / \sqrt{2}$ times (A3.16). Finally, taking into account (A2.10), the standard deviation of the third and fourth Stokes 
parameters is twice the standard deviation of the real and imaginary parts of $T_{a, h \nu}$

$$
\Delta T_{3,4}=\sqrt{2} \cdot \frac{\sqrt{\left(T_{a, h}+T_{\mathrm{R}}+2 \cdot T_{\mathrm{ph}}\right) \cdot\left(T_{a, \nu}+T_{\mathrm{R}}+2 \cdot T_{\mathrm{ph}}\right)}}{\sqrt{B \cdot \tau}} .
$$

\section{ACKNOWLEDGMENT}

This paper is patent pending and has been conducted as part of the award "Passive Advanced Unit (PAU): A Hybrid L-band Radiometer, GNSS-Reflectometer and IR-Radiometer for Passive Remote Sensing of the Ocean" made under the European Heads of Research Councils and ESF EURYI Awards scheme in 2004.

\section{REFERENCES}

[1] C. T. Swift and R. E. McIntosh, "Considerations for microwave remote sensing of ocean surface salinity," IEEE Trans. Geosci. Remote Sens., vol. GRS-21, no. 4, pp. 480-491, Jul. 1983.

[2] J. Font, G. S. E. Lagerloef, D. M. Le Vine, A. Camps, and O. Zanifé, "The determination of surface salinity with the European SMOS space mission," IEEE Trans. Geosci. Remote Sens., vol. 42, no. 10, pp. 2196-2205, Oct. 2004.

[3] C. J. Koblinsky, D. Le Vine, F. Pellerano, Y. Chao, W. Wilson, S. Yueh, and G. Lagerloef, "Sea surface salinity from space: Science goals and measurement approach," Radio Sci., vol. 38, no. 4, 8064, Jun. 2003. DOI: 10.1029/2001RS002584.

[4] A. Camps, J. Font, M. Vall-llossera, C. Gabarró, I. Corbella, N. Duffo, F. Torres, S. Blanch, A. Aguasca, R. Villarino, L. Enrique, J. Miranda, J. Arenas, A. Juliá, J. Etcheto, V. Caselles, A. Weill, J. Boutin, S. Contardo, R. Niclós, R. Rivas, S. C. Reising, P. Wursteisen, M. Berger, and M. Matín-Neira, "The WISE 2000 and 2001 field experiments in support of the SMOS mission: Sea surface L-band brightness temperature observations and their application to multi-angular salinity retrieval," IEEE Trans. Geosci. Remote Sens., vol. 42, no. 4, pp. 804-823, Apr. 2004.

[5] R. Sabia, A. Camps, M. Vall-Llossera, and N. Reul, "Impact on sea surface salinity retrieval of different auxiliary data within the SMOS mission," IEEE Trans. Geosci. Remote Sens., vol. 44, no. 10, pp. 2769-2778, Oct. 2006

[6] A. Camps, I. Corbella, M. Vall-llossera, N. Duffo, F. Torres, R. Villarino, L. Enrique, J. Miranda, F. Julbé, J. Font, A. Juliá, C. Gabarró, J. Etchetto, J. Boutin, A. Weill, V. Caselles, E. Rubio, P. Wursteisen, M. Berger, and M. Martín-Neira, "L-band sea surface emissivity: Preliminary results of the WISE-2000 campaign and its application to salinity retrieval in the SMOS mission," Radio Sci., vol. 38, no. 4, 8071, 2003. DOI: 10.1029/2002RS002629.

[7] M. Martín-Neira, "A passive reflectometry and interferometry system (PARIS): Application to ocean altimetry," ESA J., vol. 17, pp. 331-355, 1993.

[8] M. Martin-Neira, M. P. Colmenarejo, G. Ruffini, and C. Serra, "Ocean altimetry using the carrier phase of GNSS reflected signals," in Proc. Ocean Winds. Plouzane, France: CERSAT Bulletin, Issue 11, Science Topic 22, Nov. 2000.

[9] M. Belmonte Rivas and M. Martin-Neira, "Coherent GPS reflections from the sea surface," IEEE Geosci. Remote Sens. Lett., vol. 3, no. 1, pp. 28-31, Jan. 2006.

[10] F. Soulat, M. Caparrini, O. Germain, P. Lopez-Dekker, M. Taani, and G. Ruffini, "Sea state monitoring using coastal GNSS-R," Geophys. Res. Lett., vol. 31, p. L21303, 2004.DOI: 10.1029/2004GL020680.

[11] G. Ruffini, M. Caparrini, B. Chapron, F. Soulat, O. Germain, and L. Ruffini, "Oceanpal: An instrument for remote sensing of the ocean and other water surfaces using GNSS reflections," in Proc. EuroGOOS, Athens, Greece, Dec. 3-6, 2002, pp. 146-153.

[12] J. L. Garrison and J. L. Katzberg, "Effects of sea roughness on bistatically scattered range coded signals from the global positioning system," Geophys. Res. Lett., vol. 25, no. 13, pp. 2257-2260, Jul. 1998.

[13] A. Komjathy, V. Zavorotny, P. Axelrad, G. H. Born, and J. L. Garrison, "GPS signal scattering from sea surface: Wind speed retrieval using experimental data and theoretical model," Remote Sens. Environ., vol. 73, no. 2 , pp. $162-174$, Aug. 2000
[14] J. L. Garrison, A. Komjathy, V. U. Zavorotny, and S. J. Katzberg, "Wind speed measurement using forward scattered GPS signals," IEEE Trans. Geosci. Remote Sens., vol. 40, no. 1, pp. 50-65, Jan. 2002.

[15] E. Cardellach, G. Ruffini, D. Pino, A. Rius, A. Komjathy, and J. L. Garrison, "Mediterranean balloon experiment: Ocean wind speed sensing from the stratosphere, using GPS reflections," Remote Sens. Environ., vol. 88, no. 3, pp. 351-362, Dec. 15, 2003.

[16] S. Lowe, C. Zuffada, Y. Chao, P. Kroger, J .L LaBreque, and L. E. Young, "5-cm precision aircraft ocean altimetry using GPS reflections," Geophys. Res. Lett., vol. 29, no. 10, pp. 4359-4362, 2002.

[17] A. Rius, J. M. Aparicio, E. Cardellach, M. Martín-Neira, and B. Chapron, "Sea surface state measured using GPS reflected signals," Geophys. Res. Lett., vol. 29, no. 23, p. 2122, Dec. 2002.

[18] G. Ruffini, F. Soulat, M. Caparrini, O. Germain, and M. Martín-Neira, "The Eddy experiment: Accurate GNSS-R ocean altimetry from low altitude aircraft," Geophys. Res. Lett., vol. 31, p. L12306, 2004.DOI: 10.1029/2004GL019994.

[19] O. Germain, G. Ruffini, F. Soulat, M. Caparrini, B. Chapron, and P. Silvestrin, "The Eddy experiment: GNSS-R speculometry for directional sea-roughness retrieval from low altitude aircraft," Geophys. Res. Lett., vol. 31, p. L21307, 2004.DOI: 10.1029/2004GL020991.

[20] S. T. Lowe, J. L. LaBrecque, C. Zuffada, L. J. Romans, L. E. Young, and G. A. George, "First spaceborne observation of an Earth-reflected GPS signal," Radio Sci., vol. 37, no. 1, 07, Feb. 2002.

[21] S. Gleason, S. Hodgart, S. Yiping, C. Gommenginger, S. Mackin, M. Adjrad, and M. Unwin, "Detection and processing of bistatically reflected GPS signals from low Earth orbit for the purpose of ocean remote sensing," IEEE Trans. Geosci. Remote Sens., vol. 43, no. 6 , pp. 1229-1241, Jun. 2005.

[22] A. Camps, M. Caparrini, R. Sabia, and G. Ruffini, "Sea surface salinity retrieval from space: Potential synergetic use of GNSS-R signals to improve the sea state correction and application to the SMOS mission," in Proc. IEEE Microrad Conf., San Juan, Puerto Rico, Feb. 28-Mar. 3, 2006, pp. 91-96.

[23] A. Camps, "Passive Advanced Unit (PAU): A hybrid L-band radiometer, GNSS-Reflectometer and IR-Radiometer for passive remote sensing of the ocean," Project descriptions of the EURYI award winners 2004, Telecommunications Engineering. [Online]. Available: http://www.esf.org/activities/euryi/awards-results/2004.html

[24] I. Ramos-Perez, X. Bosch-Lluis, A. Camps, J. F. Marchan-Hernandez, and R. Prehn"Design of a compact dual-polarization receiver for pseudocorrelation radiometers at L-band," in Proc. IGARSS, Denver, CO, Jul. 31-Aug. 4, 2006, pp. 1172-1175.

[25] X. Bosch-Lluis, A. Camps, J. F. Marchan-Hernandez, I. Ramos-Perez, and R. Prehn, "FPGA-based implementation of a polarimetric radiometer with digital beamforming," in Proc. IGARSS, Denver, CO, Jul. 31Aug. 4, 2006, pp. 1176-1179.

[26] J. F. Marchan-Hernandez, I. Ramos-Perez, X. Bosch-Lluis, A. Camps, and R. Prehn, "FPGA-based implementation of DDM-generator for GPSreflectometry," in Proc. IGARSS, Denver, CO, Jul. 31-Aug. 4, 2006, pp. $1180-1183$.

[27] S. W. Wedge and D. B. Rutledge, "Wave techniques for noise modeling and measurement," IEEE Trans. Microw. Theory Tech., vol. 40, no. 11, pp. 2004-2012, Nov. 1992

[28] J. Randa, "Noise characterization of multiport amplifiers," IEEE Trans. Microw. Theory Tech., vol. 49, no. 10, pp. 1757-1763, Oct. 2001.

[29] I. Corbella, F. Torres, A. Camps, N. Duffo, M. Vall-1lossera, K. Rautiainen, M. Martín-Neira, and A. Colliander, "Analysis of correlation and total power radiometer front-ends using noise waves," IEEE Trans. Geosci. Remote Sens., vol. 43, no. 11, pp. 2452-2459, Nov. 2005.

[30] I. Corbella, A. Camps, F. Torres, and J. Bará, "Analysis of noise injection networks for interferometric radiometer calibration," IEEE Trans. Microw. Theory Tech., vol. 48, no. 4, pp. 545-552, Apr. 2000.

[31] K. Masuda, T. Takashima, and Y. Takayama, "Emissivity of pure sea waters for the model sea surface in the infrared window regions," Remote Sens. Environ., vol. 24, pp. 313-329, 1988.

[32] A. Berk, P. K. Acharya, L. S. Bernstein, M. W. Matthew, S. M. Adler-Golden, G. P. Anderson, M. L. Hoke, J. H. Chetwynd, and E. P. Shettle, MODTRAN4 Version 3 Revision 1 USER'S MANUAL, 2003. Spectral Sciences, Inc; Air Force Research Laboratory, Space Vehicles Directorate, Air Force Materiel Command; and Naval Research Laboratory, Remote Sensing Division.

[33] M. Bersanelli, N. Mandolesi, S. Weinreb, R. Ambrosini, and G. F. Smoot, "The LFI differential receiver concept," Internal Report ITESRE 177/1995, Mar. 1995. COBRAS memo n. 5.

[34] F. Torres, A. Camps, J. Bará, I. Corbella, and R. Ferrero, "On-board phase and module calibration of large apertura síntesis radiometer. Study 
applied to MIRAS," IEEE Trans. Geosci. Remote Sens., vol. 34, no. 4, pp. 1000-1009, Jul. 1996.

[35] S. Ribó, M. Martín-Neira, and F. Torres, "Faraday rotation correction using the polarimetric brightness temperature matrix," in Proc. 8th MicroRad, Univ. La Sapienza, Feb. 2004.

[36] J. W. Goodman, Statistical Optics. New York: Wiley-Interscience, 1985. Section 2.8.

[37] F. T. Ulaby, R. K. Moore, and A. K. Fung, Microw. Remote Sens.: Active and Passive, vol. I. Norwood, MA: Artech House, 1981.

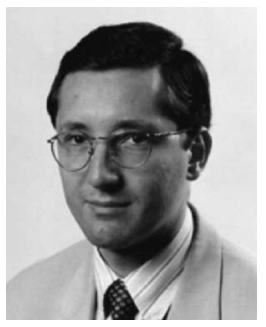

Adriano Camps (S'91-A'97-M'00-SM'03) was born in Barcelona, Spain, in 1969. He received the degree in telecommunications engineering and the Ph.D. degree in telecommunications engineering from the Universitat Politècnica de Catalunya (UPC), Barcelona, Spain, in 1992 and 1996, respectively.

In 1991-1992, he was with the Ecole Nationale Superieure des Télécommunications de Bretagne, France, with an Erasmus Fellowship. In 1993, he was with the Electromagnetics and Photonics Engineering Group at the Department of Signal Theory and Communications at UPC, as Assistant Professor, and since 1997 as Associate Professor. In 1999, he was on sabbatical leave at the Microwave Remote Sensing Laboratory, University of Massachusetts, Amherst. His research interests are focused on microwave remote sensing, with special emphasis in microwave radiometry by aperture synthesis techniques. He is Associate Editor of Radio Science.

Dr. Camps has performed numerous studies within the frame of European Space Agency SMOS Earth Explorer Mission that have received several awards. In 1993, he received the second national award of university studies; in 1997, the INDRA award of the Spanish Association of Telecommunication Engineering to the best Ph.D. in Remote Sensing; in 1999, the extraordinary Ph.D. award at UPC; in 2002, the Research Distinction of the Generalitat de Catalunya for contributions to microwave passive remote sensing; and in 2004, he received a European Young Investigator award. Also, as a member of the Microwave Radiometry Group at UPC, he received, in 2000, 2001, and 2004: the 1st Duran Farell and the Ciudad de Barcelona awards for Technology Transfer, and the "Salvst I Campillo" Award of the Professional Association of Telecommunication Engineers of Catalonia for the most innovative research project. He was Chair of $\mu \mathrm{Cal}$ 2001. From 2003 to 2006, he was Editor of the IEEE Geoscience AND REMOTE SENSing Newsletter, and President-Founder of the IEEE Geoscience and Remote Sensing Society Chapter at Spain.

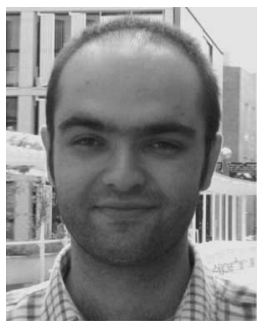

Xavi Bosch-Lluis received the degree in telecommunication engineering from the Universitat Politècnica de Catalunya, Barcelona, Spain, in May 2005.

He was with the Passive Remote Sensing Group at the Department of Signal Theory and Communications. His current research activities are related to L-band radiometry, digital beamforming, calibration techniques, and reflectometry of GNSS-R.

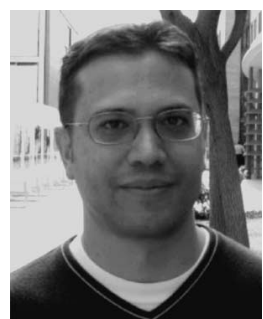

Isaac Ramos-Pérez (S'04-A'06) was born in Barcelona, Spain. He received the degree in telecommunications engineering from the Universitat Politècnica de Catalunya (UPC), Barcelona, Spain, in May 2005, where he is currently working toward the Ph.D. degree in microwave radiometry, developing a synthetic aperture passive advanced unit, a new instrument to develop and test potential improvements for the future SMOS Operational System.

He was with Passive Remote Sensing Group at the Department of Signal Theory and Communications.

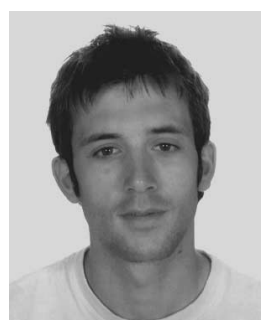

Juan Fernando Marchán-Hernández (S'04) was born in Barcelona, Spain. He received the M.S. degree from the Polytechnic University of Catalonia (UPC), Barcelona, in 2004.

He was with the Passive Remote Sensing Group of the Department of Signal Theory and Communications, UPC. In 2003, he was with the Laboratory of Space Technology, Helsinki University of Technology (TKK). His current research activities are related to radiometry and signal reflections of GNSS-R.

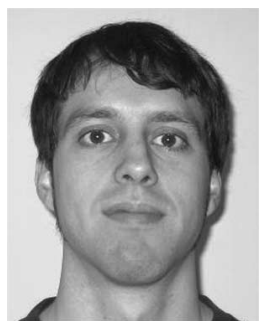

Benjamín Izquierdo received the degree in telecommunication engineering from the Polytechnic University of Catalonia, Barcelona, Spain, in 2006, where he is currently working toward the Ph.D. degree.

His main research interests deal with microwave radiometry and electromagnetic propagation.

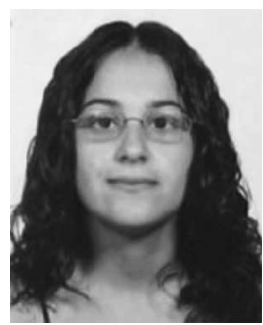

Nereida Rodríguez-Álvarez (S'07) was born in El Prat de Llobregat, Barcelona, Spain. She is currently working toward the degree in telecommunication engineering at the Universitat Politècnica de Catalunya, Barcelona, where she is performing her final project at the Passive Remote Sensing Group.

Her current research activities are related to infrared radiometry. 